\title{
Implementation of adaptive fault-tolerant tracking control for robot manipulators with integral sliding mode
}

\author{
Linzhi Liu • Liyin Zhang • Yinlong \\ Hou • Dafeng Tang • Hui Liu
}

Received: date / Accepted: date

\begin{abstract}
This paper addresses an adaptive fault-tolerant tracking control for robot manipulators. By fully considering the effects of uncertainties and actuator effectiveness faults (UAEFs), a robust fault-tolerant tracking control combining with an auxiliary function and an integral sliding manifold is first developed for uncertain robot manipulators. Then, an adaptive law for unknown parameters of the upper bounded uncertainties is constructed to obtain a robust fault-tolerant approach with the elimination of the reaching phase of sliding mode control (SMC). The stability of the proposed approaches is accomplished by Lyapunov stable theory. The key contributions of the proposed approach are as follows: i) the reaching phase of SMC is removed in the control design and then the sliding mode starts at very beginning; (ii) the nominal control term is eliminated in the design of integral sliding surface and then the algebraic loop problem is also avoided in the proposed approach for robot manipulators; (iii) the simple control structure with an adaptive law is obtained for improving chattering-restraining ability of the proposed approach and then the effects of time delay and computational burden are also restrained from the proposed approach. Simulation and experimental comparisons have been accomplished for verifying the effectiveness of the proposed approach.
\end{abstract}

Keywords Fault-tolerant control · Sliding mode control · Robot manipulators $\cdot$ Adaptive control

Liyin Zhang · Yinlong Hou · Dafeng Tang · Hui Liu

Xi'an Key Laboratory of Advanced Control and Intelligent Process, School of Automation, Xi'an University of Posts and Telecommunications, Xi'an, China

E-mail: zhangliyin@xupt.edu.cn

Linzhi Liu

China State Shipbuilding Corporation Limited, Xi'an Dong Yi Science Technology and Industry Group Co., Ltd, Xi'an, China 


\section{Introduction}

Inspiring by high efficiency, high reliability, low cost and high precision, robot manipulators have widely been utilized in various industrial application fields such as drilling, welding and painting [1-9]. In particular, the safety of robot manipulators is gradually promoted on an important place with the increased control precision requirements. The faults of robot manipulators derive mainly from two aspects including the sensor faults and the actuator faults, which not only reduce the control precision, but also threaten human safety. Among them, the actuator faults have a great impact on the tracking performance of robot manipulators than sensor faults and thus attracted more attention in recent research results. In additions, uncertain dynamics and external disturbances (UDEDs) are another kind of factors affecting the control performance of robot manipulators. Accordingly, it is still a challenging issue to develop a robust control of robot manipulators subject to uncertainties and actuator effectiveness faults (UAEFs).

In general, the actuator faults are offer eliminated by a fault-tolerant tracking control (FTC) with/without the faults observer [10,11]. As a result, FTCs are always decomposed into the passive FTC (PFTC) and active FTC (AFTC). For AFTC, the actuator faults are obtained by an fault observer $[12,13]$, but the calculated amount of controller will be increased vastly [14]. On the contrary, the actuator effectiveness faults (AEF) for PFTC are determined as uncertainties of robot manipulators. Meanwhile, the PFTC does not claim the feedback of faults observer and indeed decreases the computational burden of controller. In conclusion, the PFTC guarantees rapid transient response than the active one, but employs larger control input than the active one [15]. In addition, the conventional FTCs are sensitive to the UDEDs of robot manipulators. With the strong robustness to the matched uncertainties, sliding mode controls (SMC) are always injected into the FTCs to overcome the effects of UAEFs on tracking performance $[16,17]$. Upon the advantages of time-delay estimation and backstepping techniques, Mien et al. $[18,19]$ propose a novel SMC for the finite-time stable FTC systems. However, these approaches [16-19] neglect the effects of the algebraic loop problem on tracking performance of robot manipulators, in which the joint acceleration or its upper bound is involved into the control design. In the applications of robot manipulators, the algebraic loop problem not only amplifies the control torque input but also destroys the stability of robot tracking system [20]. Accordingly, the following drawbacks still limit the application of SMC for FTC systems. They are as follows: (i) it brings the algebraic loop problem [20], which enlarges the control torque input and destroys the stability of controller system; (ii) it requires the reaching phase of SMC, which decreases the tracking precision and enlarges the amplitude of the chattering; (iii) it requires the upper bounds of uncertainties of robot manipulators. In order to obtain the SMCs without the reaching phase, several significant works related to integral SMC (ISMC) have been developed in [21-24]. 
The ISMC is classified into two categories, i.e., the ISMC with/without the reaching phase [25-28]. A nonlinear integral-type SMC without involving the reaching phase is proposed in [21] for both matched and unmatched uncertain systems, which give rise to a common definition of ISMC along with its stability analysis. The ISMC without the reaching phase reveals enormous advantages in both tracking precision and chattering suppression [29]. Based on these seminal works $[21,29]$, the ISMCs for FTC system have been introduced in [30-33] to the practical applications of various nonlinear systems. In particular, Ref. [30] provides an ISMC for FTC of a linear time-invariant system; while the detailed analysis of ISMC for a nonlinear FTC system is proposed in [31]. In [32] and [33], the ISMCs are further developed in the control formulation of attitude FTC of spacecraft. By utilizing a fuzzy logic system, Mien Van et al. [34] proposed an ISMC for FTC of robot manipulators, in which the disturbance observer is introduced to estimate the unknown nonlinear terms. These approaches are of great significance to the development of ISMCs uninvolved the reaching phase. However, in practical applications of robot manipulators above ISMCs [21,29-33] without the reaching phase ignore several facts as follows: (i) the integral sliding surface includes the nominal control input, which may cause the new algebraic loop problem [20]; (ii) the stability analysis on sliding phase is not provided in these existing approaches.

In addition, the undesired chattering is another limitation of the ISMC/SMC applications for robot manipulators. It is well-known that the magnitude of controller chattering is proportional to the control gain which is commonly chosen as a larger value than the upper bound of uncertainties. Several approaches such as the boundary method [30], the neural network, the fuzzy logic system and adaptive SMC [35-42], have been developed to reduce the effects of chattering phenomenon of SMC/ISMC. In these approaches, the chatteringrestraining is mainly divided into two categories, i.e., the adaptive estimation of the control gain related to the upper bound of uncertain [43-46] and the whole estimation of the lumped uncertainties. Among them, the neural network and the fuzzy logic techniques $[6-8,35,36]$ is always acted as the whole estimation of the lumped uncertainties for SMC/ISMC of robot manipulators. Nevertheless, these approaches [35-39] bring great tracking performance and meanwhile introduces several limitations such as high time-delay, large calculated amount and complex control structure.

From above overview, there still exists several factors which limits the practical application of ISMC for robot manipulators. These factors are as follows: (i) the algebraic loop problem should be further eliminated; (ii) the nominal control term should be removed in the design of integral sliding manifold; (iii) the stability analysis on sliding phase needs to be further proved. Inspired by the works $[21,34,47]$, in this paper we have focused on the development of robust ISMC uninvolved the reaching phase for an FTC of robot manipulators by combining an ISMC and an adaptive technique. First, an FTC approach for robot manipulators is developed by combining an ISMC subject to UAEFs. In our design, a novel integral sliding manifold is first designed in the formulation of the proposed approach, in which the reaching phase of an ISMC is 
removed completely and meanwhile the nominal control term is eliminated in the formulation of the integral sliding manifolds. Since the sliding mode exists from very beginning, the FTC system based on an ISMC has more robustness against uncertainties and actuator faults than other SMC/ISMC with reaching phase. Restricted by the inherent properties, the chattering phenomenon still affects the applications and robustness of SMC. Second, an adaptive law is employed to estimate unknown parameters of the upper bound of the lumped uncertainties, which greatly reduces the magnitude of actuator chattering. Finally, the simulation and experimental results have been accomplished on two-DOFs robot manipulators to verify the superior performance of the proposed approach in comparison with other state-of-the-art controllers for the FTC systems. In summary, the contributions of the proposed approach are as follows

(i) In our design, the singularity and algebraic loop problem [20] are removed completely in the proposed approach; meanwhile, the upper bounds of the UAEFs are eliminated in the control design.

(ii) Distinguished from the works [21,29-34], the proposed integral sliding manifold removes the nominal control term in the proposed ISMC design and then the algebraic loop and computation complexity are greatly reduced. In addition, the stability analysis of ISMC uninvolved the reaching phase on sliding phase has been accomplished completely.

(iii) An adaptive technique is developed to assess unknown parameters of the upper bounded uncertainties. As a result, the chattering-restraining ISMC system is developed for eliminating the chattering amplitude of control input for uncertain robot manipulators.

Based on the meaningful key points, the proposed approach has some advantages over other ISMCs for FTC systems, such as simple control structure, chattering-restraining, rapid transient response, high steady-state tracking precision and lower control efforts.

\section{Preliminaries}

To benefit for the following control formulation, the homogeneity definition and some Lemmas for robot manipulators are firstly introduced.

Definition 1. For a proper positive definite function $V: \Re^{n} \rightarrow \Re$, if $V\left(v^{r_{1}} z_{1}, \ldots, v^{r_{n}} z_{n}\right)=v^{k} V(z), \forall z \in \Re^{n}$ where an arbitrary positive constant $v>0$ and $r=\left(r_{1}, \ldots, r_{n}\right) \in \Re_{+}^{n}, V$ is homogeneous of degree $k$. If the $f_{i}$ is a homogeneous function of degree $r_{i}+k$ for all $1 \leq i \leq n$, then the vector $f=\left[f_{1}, \ldots, f_{n}\right]^{T}$ is homogeneous of degree $k$.

\subsection{Robot Manipulators and Properties}

Consider the following robot manipulators with $n$-DOFs as [48]

$$
\bar{M}(q) \ddot{q}+\bar{C}(q, \dot{q}) \dot{q}+\bar{G}(q)=\bar{\Gamma} \bar{\tau}+\bar{d}
$$


where $q, \dot{q}, \ddot{q} \in \Re^{n}$ define the joint position, velocity and acceleration vectors, respectively, $\bar{M}(q), \bar{C}(q, \dot{q}) \in \Re^{n \times n}$ represent the symmetric positive definite inertia matrix and the centrifugal-Coriolis matrix, respectively, $\bar{G}(q) \in \Re^{n}$ stands for the gravitational torque vector, $\bar{d} \in \Re^{n}$ is the bounded external disturbances satisfying the condition $\|\bar{d}\| \leq \bar{d}_{m}$ with a known constant $\bar{d}_{m}$, $\bar{\tau} \in \Re^{n}$ denotes the control input, $\bar{\Gamma}=\operatorname{diag}\left\{\bar{\gamma}_{i}(t)\right\}, i=1, \ldots, n$ stands for the actuator health condition with $\bar{\gamma}_{0} \leq \bar{\gamma}_{i}(t) \leq 1$, and $\bar{\gamma}_{0} \in(0,1]$ represents a known positive constant.

Assumption 1. The robot manipulators model (1) is modified [48]

$$
\begin{gathered}
\bar{M}(q)=\bar{M}_{0}(q)+\Delta \bar{M}(q) \\
\bar{C}(q, \dot{q})=\bar{C}_{0}(q, \dot{q})+\Delta \bar{C}(q, \dot{q}) \\
\bar{G}(q)=\bar{G}_{0}(q)+\Delta \bar{G}(q)
\end{gathered}
$$

where $\bar{M}_{0}(q), \bar{C}_{0}(q, \dot{q})$ and $\bar{G}_{0}(q)$ represent the known dynamics, and $\Delta \bar{M}(q)$, $\Delta \bar{C}(q, \dot{q})$ and $\Delta \bar{G}(q)$ illustrate uncertainties.

To benefit for subsequent control development and stability analysis, the following vectors have been illustrated.

$$
\begin{gathered}
\operatorname{Sgn}(\kappa)=\left[\operatorname{sign}\left(\kappa_{1}\right), \ldots, \operatorname{sign}\left(\kappa_{n}\right)\right]^{T} \\
\operatorname{Sig}^{\alpha}(\kappa)=\left[\operatorname{sig}^{\alpha}\left(\kappa_{1}\right), \ldots, \operatorname{sig}^{\alpha}\left(\kappa_{n}\right)\right]^{T}
\end{gathered}
$$

where $\alpha>0, \operatorname{sig}^{\alpha}\left(\kappa_{i}\right)=\left|\kappa_{i}\right|^{\alpha} \operatorname{sign}\left(\kappa_{i}\right)$ with $\kappa_{i}$ represents the $i$ th component of $\kappa \in \Re^{n}$, and $\operatorname{sign}(\cdot)$ is defined as a signum function.

The key designs are composed of two stages as follows: i) an novel integral manifold uninvolved the reaching phase with its robust controller is proposed to overcome the UAEFs; ii) by combining with an adaptive technique, the proposed approach is further developed as an adaptive FTC for robot manipulators. To this end, in this paper the errors in position and velocity tracking are represented as

$$
e=q-q_{d}, \quad \dot{e}=\dot{q}-\dot{q}_{d}
$$

\subsection{Error System Development}

Upon the results of Assumption 1 and (5), robot system (1) is further degenerated as

$$
\bar{M}_{0}(q) \ddot{e}=\bar{\Gamma} \bar{\tau}+\bar{\rho}+\bar{\eta}
$$

where the nominal part $\bar{\eta} \in \Re^{n}$ and the lumped uncertainty $\bar{\rho} \in \Re^{n}$ are given as

$$
\begin{gathered}
\bar{\rho}=-\Delta \bar{M}(q) \ddot{q}-\Delta \bar{C}(q, \dot{q}) \dot{q}-\Delta \bar{G}(q) \\
\bar{\eta}=-\bar{C}_{0}(q, \dot{q}) \dot{q}-\bar{G}_{0}(q)-\bar{M}_{0}(q) \ddot{q}_{d}
\end{gathered}
$$

In virtue of (1) and (2), we have obtained

$$
\Delta \bar{M}(q) \ddot{q}=\bar{E}(\bar{\Gamma} \bar{\tau}-\bar{C}(q, \dot{q}) \dot{q}-\bar{G}(q)+\bar{d})
$$


where $\bar{E} \in \Re^{n \times n}$ illustrated by [20] is

$$
\bar{E}=I_{n}-\bar{M}_{0}(q) \bar{M}^{-1}(q)
$$

with $I_{n}$ representing the unit matrix.

Inspired by Ref. [20], the nominal matrix $\bar{M}_{0}(q)$ is represented as

$$
\bar{M}_{0}=\frac{2}{\bar{\gamma}_{1}+\bar{\gamma}_{2}} I_{n}
$$

where $\bar{\gamma}_{1}$ and $\bar{\gamma}_{2}$ are illustrated as follows

$$
\bar{\gamma}_{1} \leq\left\|\bar{M}^{-1}(q)\right\| \leq \bar{\gamma}_{2}
$$

then $[20]$

$$
\|\bar{E}\| \leq \bar{\sigma}
$$

with $\bar{\sigma}>0$ given as

$$
\bar{\sigma}=\frac{\bar{\gamma}_{2}-\bar{\gamma}_{1}}{\bar{\gamma}_{1}+\bar{\gamma}_{2}}
$$

In light of Assumption 1 and (9), the upper bound of $\bar{\rho}$ represented by (7) is $[49]$

$$
\|\bar{\rho}\| \leq k_{0}+k_{1}\|\dot{q}\|^{2}+\bar{\sigma}\|\bar{\tau}\|
$$

where $k_{0}>0, k_{1}>0$, and $\bar{\sigma}$ is given by (14).

The proof of (15) can be found in Ref. [49].

\section{Integral Sliding Mode Control}

\subsection{Control Development}

For system (6), in this part we have aimed to develop a robust FTC with integral sliding mode (RFTC-ISM) for robot manipulators.

Firstly, the following nonlinear function is introduced as [50]

$$
\bar{f}\left(e_{i}\right)= \begin{cases}\bar{K}_{1} e_{i}+\bar{K}_{2} \operatorname{sig}^{2}\left(e_{i}\right), & \left|e_{i}\right|<\kappa \\ \operatorname{sig}^{r}\left(e_{i}\right), & \left|e_{i}\right| \geq \kappa\end{cases}
$$

with $0<r<1, \bar{K}_{1}=(2-r) \kappa^{r-1}, \bar{K}_{2}=(r-1) \kappa^{r-2}, 0<\kappa<1$ denotes an arbitrary small positive constant, $\operatorname{sig}^{r}\left(e_{i}\right)$ is given by $(4)$, and $\frac{\mathrm{d} \bar{f}\left(e_{i}\right)}{\mathrm{d} e_{i}}$ is represented as

$$
\bar{g}\left(e_{i}\right)= \begin{cases}\bar{K}_{1}+2 \bar{K}_{2}\left|e_{i}\right|, & \left|e_{i}\right|<\kappa \\ r\left|e_{i}\right|^{r-1}, & \left|e_{i}\right| \geq \kappa\end{cases}
$$

To obtain the proposed RFTC-ISM approach, an auxiliary function is defined as

$$
\mu=e+\alpha e_{s}
$$

where $\alpha>0$, and

$$
\dot{e}_{s}=\bar{F}(e), \quad e_{s}(0)=-e(0) / \alpha
$$




$$
\bar{F}(e)=\left[\bar{f}\left(e_{1}\right), \ldots, \bar{f}\left(e_{n}\right)\right]^{T}
$$

with $\bar{f}\left(e_{i}\right)$ is illustrated by $(16)$.

In virtue of (18) and (19), a novel integral sliding manifold is proposed as

$$
S=\dot{\mu}+\beta \mu_{I}
$$

with

$$
\begin{gathered}
\dot{\mu}_{I}=\operatorname{Sig}^{\gamma_{1}}(\mu)+\operatorname{Sig}^{\gamma_{2}}(\dot{\mu}) \\
\mu_{I}(0)=-\frac{\dot{\mu}(0)}{\beta}=-\frac{(\dot{e}(0)+\alpha F(e(0)))}{\beta}
\end{gathered}
$$

where $\operatorname{Sig}^{\gamma_{i}}(\cdot)$ is defined by (4), $\gamma_{2}=2 \gamma_{1} /\left(\gamma_{1}+1\right)$ with a positive constant $0<\gamma_{1}<1$.

Remark 1. Observed by (18)-(23), $S(0)=0$ and $\mu(0)=0$ are guaranteed in state space. As a result, the purpose of our work is transformed into design an ISMC approach such that the proposed integral sliding manifold (21) is stabled at the origin for all time. Then, the proposed approach removes the reaching phase of SMC systems and wiped out the reaching time. Compared with [21,29-33], the proposed integral sliding manifold (21)-(23) does not involves the nominal control term $u_{0}$ and employs a simple structure, and thus the algebraic loop problem [20] is remove completely. Above advantage is one of contribution of the proposed approach.

Then, the proposed RFTC-ISM is represented as

$$
\bar{\tau}=\bar{\tau}_{0}+\bar{\tau}_{1}+\bar{\tau}_{2}
$$

with

$$
\begin{gathered}
\bar{\tau}_{0}=-\bar{\eta}-\alpha \bar{M}_{0} \bar{H}(e) \dot{e}-\beta \bar{M}_{0} \dot{\mu}_{I}-\left(\frac{2}{\bar{\gamma}_{1}+\bar{\gamma}_{2}}\right)^{1 / 2} \vartheta b(S) \\
\bar{\tau}_{1}=-b(S) \bar{w} \\
\bar{\tau}_{2}=-\frac{1-\bar{\gamma}_{0}}{\bar{\gamma}_{0}} b(S)\left\|\bar{\tau}_{0}+\bar{\tau}_{1}\right\|
\end{gathered}
$$

where $\vartheta$ denotes a positive constant, $\bar{\eta}$ and $\dot{\mu}_{I}$ are involved from (8) and (22), respectively, $\bar{M}_{0}$ is defined by (11) and

$$
\begin{gathered}
\bar{H}(e)=\operatorname{diag}\left\{\bar{g}\left(e_{i}\right)\right\}, i=1, \ldots, n \\
b(S)= \begin{cases}\frac{S}{\|S\|}, & \|S\| \neq 0 \\
0, & \|S\|=0\end{cases} \\
\bar{w}=\frac{1}{1-\bar{\sigma}}\left(k_{0}+k_{1}\|\dot{q}\|^{2}+\bar{\sigma}\left\|\bar{\tau}_{0}+\bar{\tau}_{2}\right\|\right)
\end{gathered}
$$

and $\bar{g}\left(e_{i}\right)$ is represented in (17), $k_{0}$ and $k_{1}$ are given by (15), and $\bar{\sigma}$ is defined by $(14)$.

From (24)-(30), system (6) can be transformed as

$$
\bar{M}_{0} \ddot{e}=\bar{\tau}_{0}+\bar{\tau}_{1}+\bar{\Gamma} \bar{\tau}_{2}-\left(I_{n}-\bar{\Gamma}\right)\left(\bar{\tau}_{0}+\bar{\tau}_{1}\right)+\bar{\rho}+\bar{\eta}
$$


Remark 2. Since the sliding mode of the proposed approach (24)-(30) starts at very beginning of trajectory tracking, then the strong robustness and high tracking precision have been guaranteed [29]. The reason behind this is that the state trajectory will not switched on the integral sliding manifold frequently at very beginning but stabled at the integral sliding surface to the origin for all time.

Remark 3. Observed by (24)-(30), their upper bounds $\left\|\bar{\tau}_{1}\right\|$ and $\left\|\bar{\tau}_{2}\right\|$ can not be included in the design of control parts $\bar{\tau}_{1}$ and $\bar{\tau}_{2}$ given by (26) and (27), respectively. As a result, the algebraic loop problem [20] is removed in the sliding mode tracking system of uncertain robot manipulators. Give full consideration to uncertain robot manipulators, the proposed approach reserves a simple control structure and advanced convergence property of ISMC system than other ISMC $[21,29-33]$ to implement the trajectory tracking.

\subsection{Stability Analysis}

In this subsection, the stability analysis and discussion have been accomplished.

Theorem 1. For system (31), the designed controller (24)-(30) and the proposed integral sliding manifold (21), the errors in position and velocity tracking converge globally to zero with the finite-time stability. First, the tracking trajectories are still restrained on the sliding manifold (21) excluding the reaching phase for all time, i. e., $S=0$ if $t>0$ and $S(0)=0$. Second, an auxiliary function $\mu, e$ and $\dot{e}$ along with the proposed sliding manifold (21) are guaranteed to zero with finite-time stability.

Proof. First, there exists a Lyapunov function as

$$
V=\frac{1}{2} S^{T} M_{0} S
$$

Then, the time differential of $V$ is

$$
\begin{aligned}
\dot{V}= & S^{T} M_{0} \dot{S} \\
= & S^{T} M_{0}\left(\ddot{\mu}+\beta \dot{\mu}_{I}\right) \\
= & S^{T} M_{0}\left(\ddot{e}+\alpha \ddot{e}_{s}+\beta \dot{\mu}_{I}\right) \\
= & S^{T}\left(\bar{\tau}_{0}+\bar{\tau}_{1}+\bar{\Gamma} \bar{\tau}_{2}-\left(I_{n}-\bar{\Gamma}\right)\left(\bar{\tau}_{0}+\bar{\tau}_{1}\right)+\bar{\rho}+\bar{\eta}\right. \\
& \left.+\alpha M_{0} \ddot{e}_{s}+\beta M_{0} \dot{\mu}_{I}\right)
\end{aligned}
$$

where (18), (21), (24) and (31) are utilized.

Upon substituting (25) into (33) yields

$$
\begin{aligned}
\dot{V}= & S^{T}\left(\bar{\tau}_{1}+\bar{\Gamma} \bar{\tau}_{2}-\left(I_{n}-\bar{\Gamma}\right)\left(\bar{\tau}_{0}+\bar{\tau}_{1}\right)+\bar{\rho}\right) \\
& -\left(\frac{2}{\bar{\gamma}_{1}+\bar{\gamma}_{2}}\right)^{1 / 2} \vartheta\|S\| \\
\leq & S^{T} \bar{\tau}_{1}+S^{T} \bar{\Gamma} \bar{\tau}_{2}+\|S\|\left\|I_{n}-\bar{\Gamma}\right\|\left\|\bar{\tau}_{0}+\bar{\tau}_{1}\right\| \\
& +\|S\|\|\bar{\rho}\|-\vartheta V^{1 / 2}
\end{aligned}
$$


where $V=-\frac{2}{\bar{\gamma}_{1}+\bar{\gamma}_{2}}\|S\|^{2}$ is involved from (32).

Applied (15) and (26) into (34), $S^{T} \bar{\tau}_{1}+\|S\|\|\bar{\rho}\|$ can be simplified as

$$
\begin{aligned}
S^{T} & \bar{\tau}_{1}+\|S\|\|\bar{\rho}\| \\
\leq & -\|S\| \bar{w}+\|S\|\left(k_{0}+k_{1}\|\dot{q}\|^{2}+\bar{\sigma}\|\bar{\tau}\|\right) \\
\leq & \left(k_{0}+k_{1}\|\dot{q}\|^{2}+\bar{\sigma}\left\|\bar{\tau}_{0}+\bar{\tau}_{2}\right\|\right)\|S\| \\
& \quad-\|S\| \bar{w}+\bar{\sigma}\left\|\bar{\tau}_{1}\right\|\|S\| \\
\leq & -(1-\bar{\sigma}) \bar{w}\|S\|-\bar{\sigma} \bar{w}\|S\|+\bar{\sigma}\left\|\bar{\tau}_{1}\right\|\|S\| \\
& +\left(k_{0}+k_{1}\|\dot{q}\|^{2}+\bar{\sigma}\left\|\bar{\tau}_{0}+\bar{\tau}_{2}\right\|\right)\|S\| \\
= & -\bar{\sigma} \bar{w}\|S\|+\bar{\sigma}\left\|\bar{\tau}_{1}\right\|\|S\| \\
= & 0
\end{aligned}
$$

where $\left\|\bar{\tau}_{0}+\bar{\tau}_{1}+\bar{\tau}_{2}\right\| \leq\left\|\bar{\tau}_{0}+\bar{\tau}_{2}\right\|+\left\|\bar{\tau}_{1}\right\|$ and $\left\|\bar{\tau}_{1}\right\|=\bar{w}$ are utilized from (24) and (26), respectively.

By utilizing (35) and (27), (34) can be degenerated as

$$
\begin{aligned}
\dot{V} \leq & S^{T} \bar{\Gamma} \bar{\tau}_{2}+\|S\|\left\|I_{n}-\bar{\Gamma}\right\|\left\|\bar{\tau}_{0}+\bar{\tau}_{1}\right\|-\vartheta V^{1 / 2} \\
\leq & -\left(1-\bar{\gamma}_{0}\right)\left\|\tau_{0}+\tau_{1}\right\|\|S\| \\
& +\|S\|\left\|I_{n}-\bar{\Gamma}\right\|\left\|\bar{\tau}_{0}+\bar{\tau}_{1}\right\|-\vartheta V^{1 / 2} \\
= & -\vartheta V^{1 / 2}
\end{aligned}
$$

where $\|\bar{\Gamma}\| \geq \gamma_{0}$ and $\left\|I_{n}-\bar{\Gamma}\right\| \geq 1-\gamma_{0}$ are utilized from (1).

From (18) and (21), it is observed that the proposed integral SMC (24)(30) removes the reaching phase, i. e., $S=0$ if $t>0, S(0)=0$ and $\mu(0)=0$. Combined with the result of (36), we have obtained a fact that the tracking trajectories are still restrained on the proposed sliding manifold (21) for all time (i.e., $S=0$ if $t>0$ ). The results of above analysis further verify our conclusions in Remarks 1 and 2.

Remark 4. Different from the works [21,34], another key contribution is that our design provides an detailed stability analysis on sliding phase. Thus, the subsequent contents focus on the stability analysis of ISMC on sliding phase (i.e., $S=0$ ).

If $S=0$ for all time, the integral sliding manifold (21) and (22) is further degenerated as

$$
\ddot{\mu}=\operatorname{Sig}^{\gamma_{1}}(\mu)+\operatorname{Sig}^{\gamma_{2}}(\dot{\mu})
$$

For system (37), we chosen the following Lyapunov candidate function as

$$
V_{1}=\frac{1}{1+\gamma_{1}} \sum_{i=1}^{n}\left|\mu_{i}\right|^{\gamma_{1}+1}+\frac{1}{2} \dot{\mu}^{T} \dot{\mu}
$$

By selecting the proper $\gamma_{1}$ and $\gamma_{2}$ given by (22), similar to Step 3 of [26], system (37) with $k:(\mu, \dot{\mu}) \rightarrow\left(\varsigma^{2 /\left(\gamma_{1}+1\right)} \mu, \varsigma \dot{\mu}\right)$ is homogeneous where $k=\left(\gamma_{1}-1\right) /\left(\gamma_{1}+1\right)<0$. By combining Definition 1 and the asymptotically 
stable system from (37) and (38), $\mu=0$ and $\dot{\mu}=0$ are guaranteed within a finite time.

In virtue of the results of $\mu=0$ and $\dot{\mu}=0,(18)$ is divided into Cases 1 and 2 .

Case 1. If $\left|e_{i}\right| \geq \kappa,(16),(18)$ and (21) are

$$
\begin{aligned}
& e_{i}+\alpha e_{s i}=0 \\
& \dot{e}_{s i}=\operatorname{sig}^{r}\left(e_{i}\right)
\end{aligned}
$$

The integrator (39) is degenerated as

$$
\dot{e}_{s i}=\operatorname{sig}^{r}\left(-\alpha e_{s i}\right)=-\operatorname{sig}^{r}\left(\alpha e_{s i}\right)
$$

Then, the time of $e_{s i}$ taken to the origin is obtained [45]

$$
T_{f}=\frac{\left|e_{s i}(0)\right|^{1-r}}{\alpha^{r}(1-r)}=\frac{|e(0)|^{1-r}}{\alpha(1-r)}
$$

Case 2. When $\left|e_{i}\right|<\kappa$, similarly, (16) and (18) can be further changed as

$$
\begin{gathered}
e_{i}+\alpha e_{s i}=0 \\
\dot{e}_{s i}=\bar{K}_{1} e_{i}+\bar{K}_{2} \operatorname{sig}^{2}\left(e_{i}\right)
\end{gathered}
$$

For system (42), it is further converted as

$$
\dot{e}_{s i}=-\alpha \bar{K}_{1} e_{s i}-\bar{K}_{2} \operatorname{sig}^{2}\left(\alpha e_{s i}\right)
$$

Given a Lyapunov function candidate $V_{2}=1 / 2 e_{s i}^{2}$ for (43), then its time differential is

$$
\begin{aligned}
& \dot{V}_{2}=e_{s i} \dot{e}_{s i} \\
& \quad=-\alpha \bar{K}_{1} e_{s i}^{2}-\alpha \bar{K}_{2}\left|e_{s i}\right|^{3}<0
\end{aligned}
$$

Based on Lyapunov stability and the result of (44), the states $e_{s i}$ and $e_{i}$ arrived at zero asymptotically.

Inspired by Cases 1 and 2 , the following conclusions can be obtained: i) firstly the tracking trajectories of the fault-tolerant tracking system are restrained on the proposed integral sliding manifold for all time owing to the proposed approach removes the reaching phase of SMC; ii) the auxiliary variables $\mu$ and $\dot{\mu}$ arrive at zero with finite-time stability; and iii) with a finite-time stable auxiliary function $\mu$, the position tracking errors reach to an arbitrarily small range $B_{\kappa}=\left\{e_{i}|| e_{i} \mid \leq \kappa\right\}$ with finite-time convergence, and then asymptotic stability is guaranteed.

This completes the proof. 


\section{Adaptive Fault-tolerant Tracking Control with Integral Sliding Mode}

An adaptive FTC with integral sliding mode (AFTC-ISM) scheme is further developed to estimate unknown parameters $k_{0}$ and $k_{1}$ from (15) in this section. The control law is the same as (24)-(30) except for $\bar{\tau}_{1}$. In addition, $\bar{\tau}_{1}$ and adaptive law are proposed as

$$
\bar{\tau}_{1}=-b(S) \bar{w}_{1}
$$

with

$$
\bar{w}_{1}=\frac{1}{1-\bar{\sigma}}\left(\hat{k}_{0}+\hat{k}_{1}\|\dot{q}\|^{2}+\bar{\sigma}\left\|\bar{\tau}_{0}+\bar{\tau}_{2}\right\|\right)
$$

where $\hat{k}_{0}$ and $\hat{k}_{1}$ are the estimations of the unknown parameters $k_{0}$ and $k_{1}$, and the adaptive law is

$$
\begin{gathered}
\dot{\hat{k}}_{0}=\vartheta_{0}\|S\| \\
\dot{\hat{k}}_{1}=\vartheta_{1}\|\dot{q}\|^{2}\|S\|
\end{gathered}
$$

where $\vartheta_{0}$ and $\vartheta_{1}$ stand for two estimated parameters.

For the proposed AFTC-ISM, there exists the following Theorem to guarantee the stability.

Theorem 2. For system (1) and the lumped uncertainty (7) with its upper bound (15), the parameters $k_{0}$ and $k_{1}$ are assumed to be completely unknown. For the proposed controller (24)-(29), (45) and (46) and the adaptive law (47), the tracking trajectories are still restrained on the proposed sliding manifold (21) for all time, and $e_{i}$ arrived at an arbitrarily small range $B_{\kappa}=\left\{e_{i}|| e_{i} \mid \leq \kappa\right\}$ with finite-time convergence, thereafter converges to the origin asymptotically. Proof. Given a proper Lyapunov function as

$$
V_{3}=\frac{1}{2} S^{T} M_{0} S+\sum_{i=0}^{1} \frac{1}{2 \vartheta_{i}}\left(\hat{k}_{i}-k_{i}\right)^{2}
$$

For error system $(31), \dot{V}_{3}=\frac{\mathrm{d} V}{\mathrm{~d} t}$ is

$$
\begin{aligned}
\dot{V}_{3}= & S^{T}\left(\bar{\tau}_{0}+\bar{\tau}_{1}+\bar{\Gamma} \bar{\tau}_{2}-\left(I_{n}-\bar{\Gamma}\right)\left(\bar{\tau}_{0}+\bar{\tau}_{1}\right)\right. \\
& \left.+\bar{\rho}+\bar{\eta}+\alpha M_{0} \ddot{e}_{s}+\beta M_{0} \dot{\mu}_{I}\right)+\sum_{i=0} \frac{1}{\vartheta_{i}}\left(\hat{k}_{i}-k_{i}\right) \dot{\hat{k}}_{i} \\
= & S^{T}\left(\bar{\tau}_{1}+\bar{\Gamma} \bar{\tau}_{2}-\left(I_{n}-\bar{\Gamma}\right)\left(\bar{\tau}_{0}+\bar{\tau}_{1}\right)+\bar{\rho}\right)+\left(\hat{k}_{0}-k_{0}\right)\|S\| \\
& +\left(\hat{k}_{1}-k_{1}\right)\|\dot{q}\|^{2}\|S\|-\vartheta\left(\frac{2}{\bar{\gamma}_{1}+\bar{\gamma}_{2}}\right)^{1 / 2}\|S\| \\
\leq & S^{T} \bar{\tau}_{1}+S^{T} \bar{\Gamma} \bar{\tau}_{2}+\|S\|\left\|I_{n}-\bar{\Gamma}\right\|\left\|\bar{\tau}_{0}+\bar{\tau}_{1}\right\|+\|S\|\|\bar{\rho}\| \\
& +\left(\hat{k}_{0}-k_{0}\right)\|S\|+\left(\hat{k}_{1}-k_{1}\right)\|\dot{q}\|^{2}\|S\|-\vartheta\left(\frac{2}{\bar{\gamma}_{1}+\bar{\gamma}_{2}}\right)^{1 / 2}\|S\| \\
\leq & S^{T} \bar{\tau}_{1}+\|S\|\|\bar{\rho}\|+\left(\hat{k}_{0}-k_{0}\right)\|S\| \\
& +\left(\hat{k}_{1}-k_{1}\right)\|\dot{q}\|^{2}\|S\|-\vartheta\left(\frac{2}{\bar{\gamma}_{1}+\bar{\gamma}_{2}}\right)^{1 / 2}\|S\|
\end{aligned}
$$

where $\|\bar{\Gamma}\| \geq \bar{\gamma}_{0}$ and $\left\|I_{n}-\bar{\Gamma}\right\| \geq 1-\bar{\gamma}_{0}$ are involved from system (1). 
And, $S^{T} \bar{\tau}_{1}+\|S\|\|\bar{\rho}\|$ can be degenerated as

$$
\begin{aligned}
S^{T} \bar{\tau}_{1}+\|S\|\|\bar{\rho}\| \\
\leq-\bar{w}_{1}\|S\|+\left(k_{0}+k_{1}\|\dot{q}\|^{2}+\bar{\sigma}\|\bar{\tau}\|\right)\|S\| \\
\leq-(1-\bar{\sigma}) \bar{w}_{1}\|S\|+\bar{\sigma} \bar{w}_{1}\|S\|+\left(k_{0}+k_{1}\|\dot{q}\|^{2}+\bar{\sigma}\|\bar{\tau}\|\right)\|S\| \\
\leq-\left(\hat{k}_{0}+\hat{k}_{1}\|\dot{q}\|^{2}+\bar{\sigma}\left\|\bar{\tau}_{0}+\bar{\tau}_{2}\right\|\right)\|S\| \\
\quad+\left(k_{0}+k_{1}\|\dot{q}\|^{2}+\bar{\sigma}\left\|\bar{\tau}_{0}+\bar{\tau}_{2}\right\|\right)\|S\|+\bar{\sigma}\left\|\bar{\tau}_{1}\right\|-\bar{\sigma} \bar{w}_{1}\|S\| \\
=-\left(\hat{k}_{0}-k_{0}\right)\|S\|-\left(\hat{k}_{1}-k_{1}\right)\|\dot{q}\|^{2}\|S\|
\end{aligned}
$$

Upon substituting (50) into (49), we have obtained

$$
\dot{V}_{3} \leq-\vartheta\left(\frac{2}{\bar{\gamma}_{1}+\bar{\gamma}_{2}}\right)^{1 / 2}\|S\|
$$

From (51), we have obtained a fact that $S=0$ and the estimation errors $\hat{k}_{i}-k_{i}=0$ are guaranteed in a finite time. When $S=0$, the sliding phase proof of the proposed approach can be found in (37)-(44).

Upon above stability analysis of the proposed AFTC-ISM, the tracking trajectories is still restrained on the proposed integral sliding manifold (21) for all time; then $e$ and $\dot{e}$ converge globally to zero.

This completes the proof.

Remark 5. For the proposed adaptive control (45) and (46), the proper control gains $k_{0}$ and $k_{1}$ are used for trajectory tracking of robot manipulators instead of the overestimated control parameters. Meanwhile, the simple structure (24)-(30), (45) and (46) is guaranteed to obtain similar superior tracking performance as other approaches with neural and fuzzy system [31,34], and the proposed approach further eliminates the effects of time delay and computational burden.

Remark 6. Compared with the existing ISMCs [21,29-33], the proposed approach removes the effects of the singularity and algebraic loop problem completely, and meanwhile the nominal control term is eliminated in the design of integral sliding manifold. Moreover, the strong robustness of ISMC is reserved in the proposed approach which is easy to implement with a simple control structure.

\section{Simulation example}

For the proposed AFTC-ISM, the plane robot manipulators are used to reveal the advanced tracking performance [48]. They are

$$
\begin{gathered}
\bar{M}(q)=\left[\begin{array}{cc}
\bar{W}_{1}+2 \bar{W}_{2} \cos \left(q_{2}\right) & \bar{W}_{3}+\bar{W}_{2} \cos \left(q_{2}\right) \\
\bar{W}_{3}+\bar{W}_{2} \cos \left(q_{2}\right) & \bar{W}_{4}
\end{array}\right] \\
\bar{C}(q, \dot{q})=\left[\begin{array}{cc}
-\bar{W}_{2} \sin \left(q_{2}\right) \dot{q}_{1} & -2 \bar{W}_{2} \sin \left(q_{2}\right) \dot{q}_{1} \\
0 & \bar{W}_{2} \sin \left(q_{2}\right) \dot{q}_{2}
\end{array}\right]
\end{gathered}
$$




$$
\bar{G}(q)=\left[\begin{array}{c}
\bar{W}_{5} \cos \left(q_{1}\right)+\bar{W}_{6} \cos \left(q_{1}+q_{2}\right) \\
\bar{W}_{6} \cos \left(q_{1}+q_{2}\right)
\end{array}\right]
$$

where

$$
\begin{gathered}
\bar{W}_{1}=\left(\bar{m}_{1}+\bar{m}_{2}\right) \bar{l}_{1}^{2}+\bar{m}_{2} \bar{l}_{2}^{2}+\bar{J}_{1}, \bar{W}_{2}=\bar{m}_{2} \bar{l}_{1} \bar{l}_{2}, \bar{W}_{3}=\bar{m}_{2} \bar{l}_{2}^{2} \\
\bar{W}_{4}=\bar{W}_{3}+\bar{J}_{2}, \bar{W}_{5}=\left(\bar{m}_{1}+\bar{m}_{2}\right) \bar{l}_{1} \bar{g}, \bar{W}_{6}=\bar{m}_{2} \bar{l}_{2} \bar{g}
\end{gathered}
$$

The parameters of (52)-(54) are summarized as: $\bar{m}_{1}=0.5 \mathrm{~kg}, \bar{m}_{2}=1.5 \mathrm{~kg}$, $\bar{l}_{1}=1.0 \mathrm{~m}, \bar{l}_{2}=0.8 \mathrm{~m}, \bar{J}_{1}=\bar{J}_{2}=5.0 \mathrm{~kg} \cdot \mathrm{m}$ and $\overline{\mathrm{g}}=9.8 \mathrm{~m} / \mathrm{s}^{2} . \bar{d}$ given by (1) is assumed as

$$
\bar{d}=[2 \sin (t)+0.5 \sin (200 \pi t), \cos (2 t)+0.5 \sin (200 \pi t)]^{T}
$$

The sampling period is $1 \mathrm{~ms}$. The initial states are $\left[q(0)^{T}, \dot{q}(0)^{T}\right]^{T}=$ $[1.0,1.0,0,0]^{T}$. The desired trajectories $q_{d}=\left[q_{d 1}, q_{d 2}\right]^{T}(\mathrm{rad})$ are as

$$
\begin{gathered}
q_{d 1}=1.25-7 / 5 \exp (-t)+7 / 20 \exp (-4 t) \\
q_{d 2}=1.25+\exp (-t)-1 / 4 \exp (-4 t)
\end{gathered}
$$

For the robot tracking systems, the following actuator health condition $\bar{\Gamma}$ has been considered [49]

$$
\bar{\Gamma}= \begin{cases}I_{2 \times 2}, & t<8 \mathrm{~s} \\ \operatorname{diag}\{0.7+0.01 \sin (10 t), 0.65\}, & t>8 \mathrm{~s}\end{cases}
$$

Remark 6. In the simulation and experimental comparisons, the actuator health condition (58) will be injected for verifying the fault-tolerant ability of the proposed approach. From (58), we assumed that the actuators of joints 1 and 2 can lose their $29 \%$ and $35 \%$ efficiency for the time $t>8 \mathrm{~s}$, respectively.

For the simulation comparisons, the effectiveness of the AFTC-ISM is completed over an adaptive nonsingular fast TSMC (ANFTSMC) [51] and the finite-time integral backstepping control (FIBC) [52].

FIBC [52] is represented as

$$
\begin{aligned}
& \bar{\tau}_{f i b c}=\bar{u}_{N}+\bar{u}_{A} \\
& \bar{u}_{N}=\bar{M}_{0}\left[\dot{\varphi}_{1}\left(x_{1}\right)+\varphi_{2}(z)-x_{1}+\ddot{q}_{d}\right]+\bar{C}_{0}(q, \dot{q}) \dot{q}+\bar{G}_{0}(q) \\
& \bar{\tau}_{A}=-\bar{M}_{0} \operatorname{diag}\left\{\operatorname{sign}\left(z_{i}\right)\right\} \Pi
\end{aligned}
$$

where $x_{1}=e, x_{2}=\dot{e}, z=x_{2}-\varphi_{1}\left(x_{1}\right), \Pi=\left[\hat{\chi}_{1} \ldots, \hat{\chi}_{n}\right]^{T}$ with $\hat{\chi}_{i}$ is the estimation of $\chi_{i}, \dot{\varphi}_{1}\left(x_{1}\right)=\left[\dot{\varphi}_{1}\left(x_{11}\right), \ldots, \dot{\varphi}_{1}\left(x_{1 n}\right)\right]^{T}$ gives as $\frac{\mathrm{d} \varphi_{1}\left(x_{1}\right)}{\mathrm{d} t}$ and

$$
\begin{gathered}
\dot{\hat{\chi}}_{i}= \begin{cases}0, & \text { if }\left|z_{i}\right| \leq \varepsilon \\
v_{i}\left|z_{i}\right|, & \text { if }\left|z_{i}\right|>\varepsilon\end{cases} \\
\varphi_{1}\left(x_{1}\right)=-K_{1} x_{1}-K_{4} \operatorname{Sig}^{\alpha}\left(x_{1}\right) \\
\varphi_{1}(z)=-K_{2} z-K_{5} \operatorname{Sig}^{\alpha}(z)-K_{3} z \varpi^{2}-K_{6} z \varpi \operatorname{Sig}^{\alpha}(\varpi)-\varpi \\
\dot{\varphi}_{1}\left(x_{1 i}\right)= \begin{cases}-k_{1 i} x_{2 i}-k_{4 i}\left|x_{1 i}\right|^{\alpha-1} x_{2 i}, & \left|x_{2 i}\right| \geq \varsigma \text { and } x_{2 i} \neq 0 \\
-k_{1 i} x_{2 i}-k_{4 i}\left|\Delta_{i}\right|^{\alpha-1} x_{2 i}, & \left|x_{2 i}\right|<\varsigma \text { and } x_{2 i} \neq 0 \\
0, & x_{2 i}=0\end{cases}
\end{gathered}
$$


where $\dot{\varpi}=z, 0<\alpha<1, K_{i} \in \Re^{n \times n}, i=1, \ldots, 6, v_{i}, \varepsilon, \varsigma, \Delta_{i} \in \Re^{+}$, and $k_{1 i}$ and $k_{4 i}$ represent the $i$ th components of the matrixes $K_{1}$ and $K_{4}$, respectively.

While ANFTSMC [51] is described as

$$
S=e+k_{1} \operatorname{Sig}^{\alpha}(e)+k_{2} \operatorname{Sig}^{\beta}(\dot{e})
$$

where $k_{1}>0, k_{2}>0,1<\beta<2$ and $\alpha>\beta$, and $\bar{\tau}$ is given

$$
\begin{aligned}
\bar{\tau}= & \bar{u}_{e q}+\bar{u}_{a s w} \\
\bar{u}_{e q}= & \bar{M}_{0}(q) \ddot{q}_{d}+\bar{C}_{0}(q, \dot{q}) \ddot{q}_{d}+\bar{G}(q) \\
& \quad-\frac{\bar{M}_{0}(q)}{\beta k_{2}} \operatorname{diag}\left\{\left|\dot{e}_{i}\right|^{2-\beta}\right\}\left(I_{n}+\alpha k_{1} \operatorname{diag}\left\{\left|e_{i}\right|^{\alpha-1}\right\}\right) \operatorname{Sgn}(\dot{e}) \\
\bar{u}_{a s w}= & -\bar{M}_{0}(q)\left[\Lambda S+\left(\hat{c}_{0}+\hat{c}_{1}\|q\|+\hat{c}_{2}\|\dot{q}\|^{2}+\eta\right) \operatorname{Sgn}(S)\right]
\end{aligned}
$$

with $\Lambda \in \Re^{n \times n}, \eta>0, \hat{c}_{0}, \hat{c}_{1}$ and $\hat{c}_{2}$ give the estimations of $c_{0}, c_{1}$ and $c_{2}$, respectively, which are updated by

$$
\begin{aligned}
& \dot{\hat{\hat{c}}}_{0}=\lambda_{0}\|S\|\|\dot{e}\|^{\beta-1} \\
& \dot{\hat{\hat{c}}}_{1}=\lambda_{1}\|S\|\|\dot{e}\|^{\beta-1}\|q\| \\
& \dot{\hat{\hat{c}}}_{2}=\lambda_{2}\|S\|\|\dot{e}\|^{\beta-1}\|\dot{q}\|^{2}
\end{aligned}
$$

where $\lambda_{i}>0, i=0,1,2$.

Note that $\bar{M}_{0}(q), \bar{C}_{0}(q, \dot{q})$, and $\bar{G}_{0}(q)$ given by (59)-(65) have been obtained by replacing $\bar{m}_{1}$ and $\bar{m}_{2}$ of (52)-(54) to $\hat{\bar{m}}_{1}=0.4 \mathrm{~kg}$ and $\hat{\bar{m}}_{2}=1.2 \mathrm{~kg}$. And, the constant matrix $\bar{M}_{0}$ of the proposed AFTC-ISM is defined by (11). The parameters of ANFTSMC and FIBC have the same values as the works [51] and [52], respectively. The parameters of ANFTSMC, FIBC and the proposed AFTC-ISM are given by Table 1 .

Table 1 Controller parameters selection

\begin{tabular}{lc}
\hline Controllers & Parameters \\
\hline \multirow{2}{*}{ AFTC-ISM } & $\vartheta_{0}=0.01, \bar{\gamma}_{2}=0.2, \kappa=0.1, r=0.5, \alpha=3, \beta=7$ \\
& $\vartheta_{1}=0.02, \gamma_{0}=0.65, \bar{\gamma}_{1}=0.09, \gamma_{1}=0.5$ \\
\hline \multirow{2}{*}{ FIBC } & $K_{1}=K_{2}=K_{3}=\operatorname{diag}\{3,3\}, \chi_{i 0}=[2 ; 2], \alpha=0.8, \varpi_{0}=[0 ; 0]$ \\
& $K_{4}=K_{5}=K_{6}=\operatorname{diag}\{1,1\}, \varepsilon=\varsigma=0.05, v_{i}=0.01$ \\
\hline \multirow{2}{*}{ ANFTSMC } & $\alpha=1.5, \beta=5 / 3, \eta=0.1, k_{1}=1, k_{2}=2$, \\
& $\Lambda=150, \lambda_{0}=\lambda_{1}=\lambda_{2}=0.01$ \\
\hline
\end{tabular}

In Fig. 1, the real position tracks the desired trajectories in both joint 1 and 2 correctly, which reveals a better tracking performance. Fig. 2 represents the sliding surfaces of ANFTSMC and the proposed AFTC-ISM. From (18)(23), the sliding manifold of the proposed AFTC-ISM started from zero (i.e., $S(0)=0$ ) has been still stabled at the origin for all time; meanwhile, Fig. 2 gives the same result that the proposed AFTC-ISM can removes the reaching phase of conventional SMC and then enhances the transient and steady-state convergence performance. In Fig. 2, the sliding manifold (21) has been stabled 

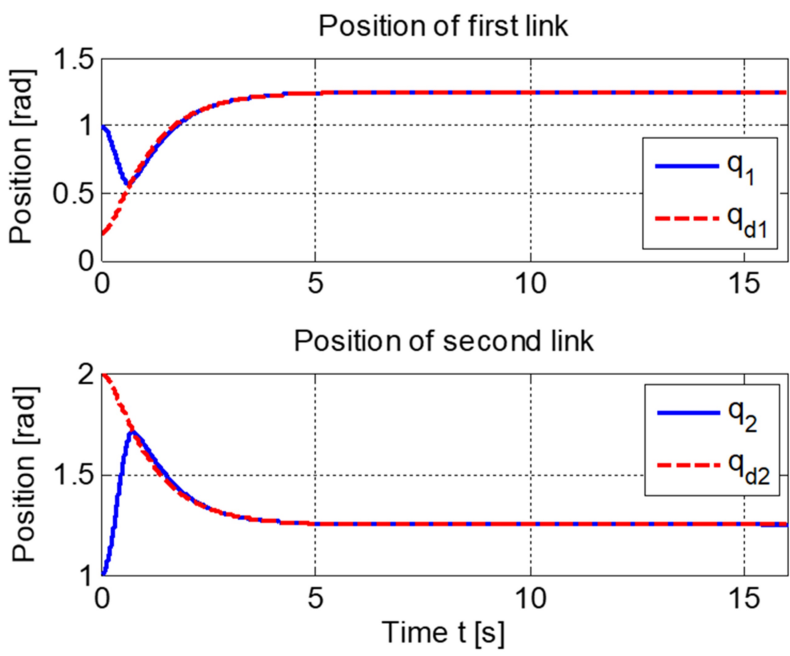

Fig. 1 Position of AFTC-ISM.
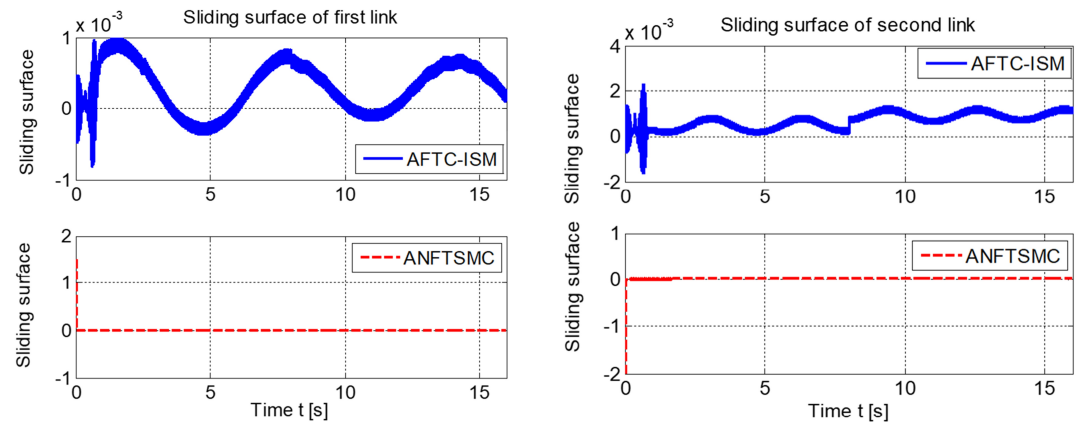

Fig. 2 Sliding surface of AFTC-ISM and ANFTSMC.
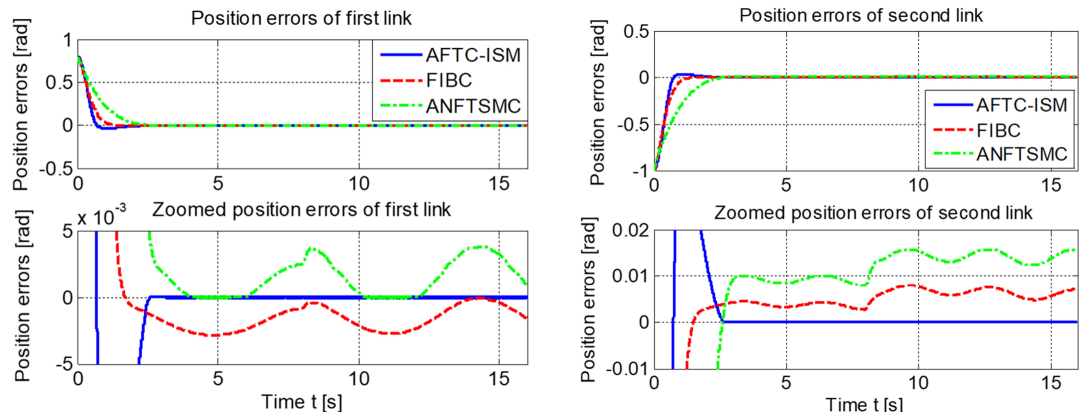

Fig. 3 Position tracking errors of AFTC-ISM, FIBC and ANFTSMC. 
at the origin with the steady-state tracking errors $1 \times 10^{-3}$ and $2 \times 10^{-3}$ for two joints, respectively. In contrast, the ANFTSMC has a larger initial value for the sliding manifold. Figs. 3 and 4 give the errors in position and velocity tracking of ANFTSMC, FIBC and the AFTC-ISM with their zoomed plots. Obviously, the proposed AFTC-ISM employs a better steady-state tracking precision than ANFTSMC and FIBC. Then, the position tracking errors of the AFTC-ISM are stabled at $5 \times 10^{-3}(\mathrm{rad})$ and $10 \times 10^{-3}(\mathrm{rad})$ for joints 1 and 2 , respectively, which provides higher steady-state tracking errors than the ANFTSMC and FIBC. While for the velocity tracking errors given by Fig. 4, the proposed AFTC-ISM provides the improved fault-tolerant ability than FIBC and ANFTSMC when the actuators lose their partial effectiveness at $t>$ $8 \mathrm{~s}$. In instant time $8 \mathrm{~s}$, the proposed AFTC-ISM reveals a better insensitivity to the AEFs. Fig. 5 gives the control input torque of FIBC, ANFTSMC and the proposed AFTC-ISM. In Fig. 5, we have obtained a fact that such favour results of the proposed approach given by Figs. 1-4 does not use the excessive control efforts than FIBC and ANFTSMC.

Remark 7. In comparison with Figs. 5, 6, 11, 12, 17 and 18 of the work [41], the proposed approach in Fig. 2 reveal a better tracking performance of integral sliding manifold. The sliding manifold of the proposed approach is still stabled
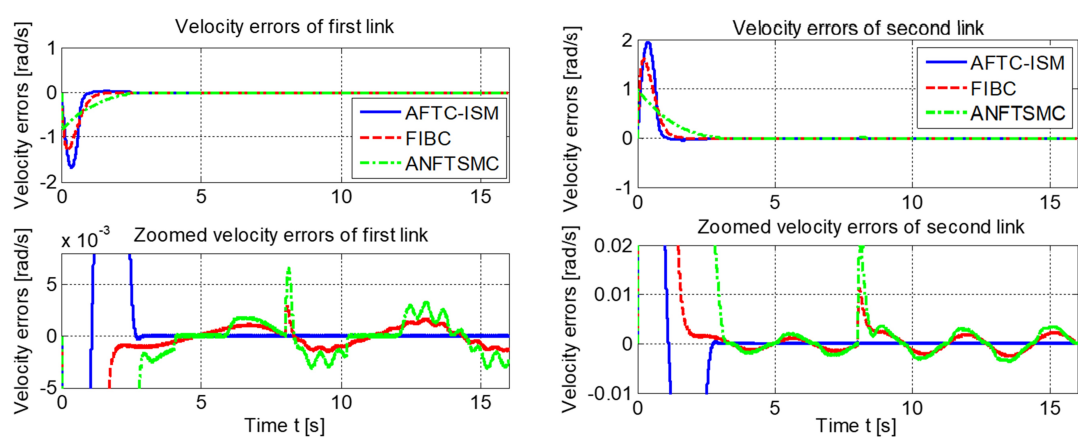

Fig. 4 Velocity tracking errors of AFTC-ISM, FIBC and ANFTSMC.
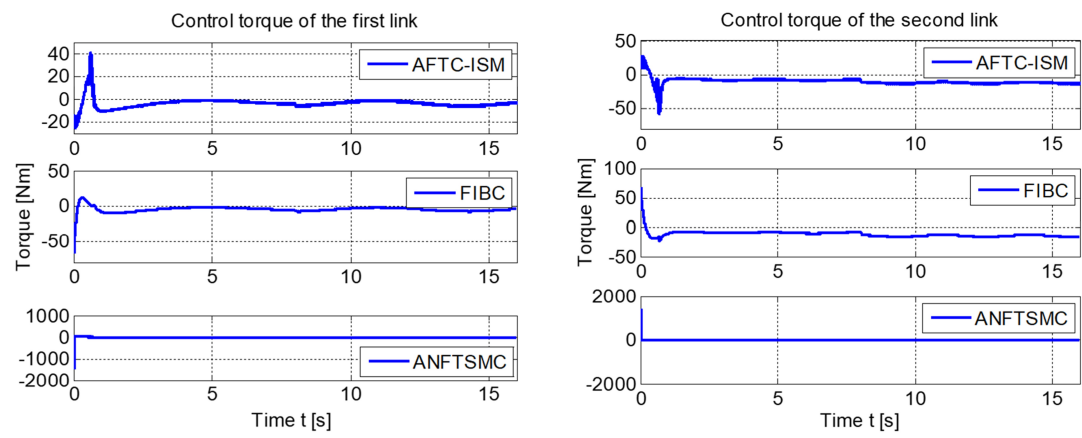

Fig. 5 Control torque input of AFTC-ISM, FIBC and ANFTSMC. 
at the orgin within a small amplitude oscillation (i.e., $1 \times 10^{-3}$ and $4 \times 10^{-3}$ for joint 1 and 2). In spite of the integral sliding manifold of [41] does not be related to the reaching phase, but it has a large overshoot of the integral sliding manifold than the proposed approach.

To further give the quantitative comparisons between the proposed approach and other controller, the following tracking precision and efforts have been accomplished after $3 \mathrm{~s}$ at the beginning of the simulation comparisons. These comparison indexes are as follows

$$
\begin{aligned}
& \bar{E}_{p}=\sqrt{\frac{1}{N} \sum_{i=1}^{N}\|e(n)\|^{2}}, \bar{E}_{v}=\sqrt{\frac{1}{N} \sum_{i=1}^{N}\|\dot{e}(n)\|^{2}} \\
& \bar{E}_{s}=\sqrt{\frac{1}{N} \sum_{i=1}^{N}\|S(n)\|^{2}}, \bar{E}_{\tau}=\sqrt{\frac{1}{N} \sum_{i=1}^{N}\|\bar{\tau}(n)\|^{2}}
\end{aligned}
$$

where $N$ stands for the total sampling number, $\bar{\tau}(n), e(n), S(n)$ and $\dot{e}(n)$ denote the control input, the position tracking errors, the sliding manifold and the velocity tracking errors at the $n$th sampling instant. These four performance indexes are illustrated in Table 2 .

Table 2 Comparison of control performance

\begin{tabular}{lcccc}
\hline Controller & $\bar{E}_{p}$ & $\bar{E}_{v}$ & $\bar{E}_{S}$ & $\bar{E}_{\tau}$ \\
\hline AFTC-ISM & $1.7 \times 10^{-3}$ & $5.7 \times 10^{-3}$ & $9.16 \times 10^{-4}$ & 19.78 \\
ANFTSMC & $1.37 \times 10^{-2}$ & $2.12 \times 10^{-2}$ & $1.39 \times 10^{-2}$ & 32.06 \\
FIBC & $5.7 \times 10^{-3}$ & $10.5 \times 10^{-3}$ & - & 20.38 \\
\hline
\end{tabular}

From Table 2, the quantitative comparisons further verify the advanced convergence performance of the proposed approach. Owing to the AFTC-ISM removes the reaching phase of SMC, the trajectories are still stabled at the sliding manifold for all time. Observed by $\bar{E}_{s}$ of Table 2, the proposed AFTC-ISM also show a higher steady-state precision of sliding manifold over ANFTSMC. As a result, the errors of the proposed AFTC-ISM without utilizing the excessive control input $\bar{E}_{\tau}$, still are stabled at a lower range than ANFTSMC and FIBC. The quantitative comparisons of Table 2 further testify the improved tracking performance of the proposed AFTC-ISM.

Remark 8. By combining the comparison results of Figs. 1-5 and Table 2, the proposed approach reveals the strong robustness than other controller no matter whether the robot tracking system is affected by the UAEFs or not. With the advantages of ISMC, the proposed approach is insensitive to matched uncertainties and actuator effectiveness faults. In addition, such favour results have been accomplished by a simple control structure. 


\section{Experimental Comparison}

In this section we have accomplished by the improved tracking performance on the SCARA robot plantform shown in Fig. 6. For this experimental platform, the maximum actuators of two joints are summarized as $[ \pm 51.2, \pm 16]^{T}(\mathrm{Nm})$ with the harmonic reduction ratio as 1 : 80 and 1 : 50, respectively. The torques are calculated though the high performance computer of the simulink of Matlab 2012b. The parameters of ANFTSMC, FIBC and the proposed AFTC-ISM are described in Table 3.

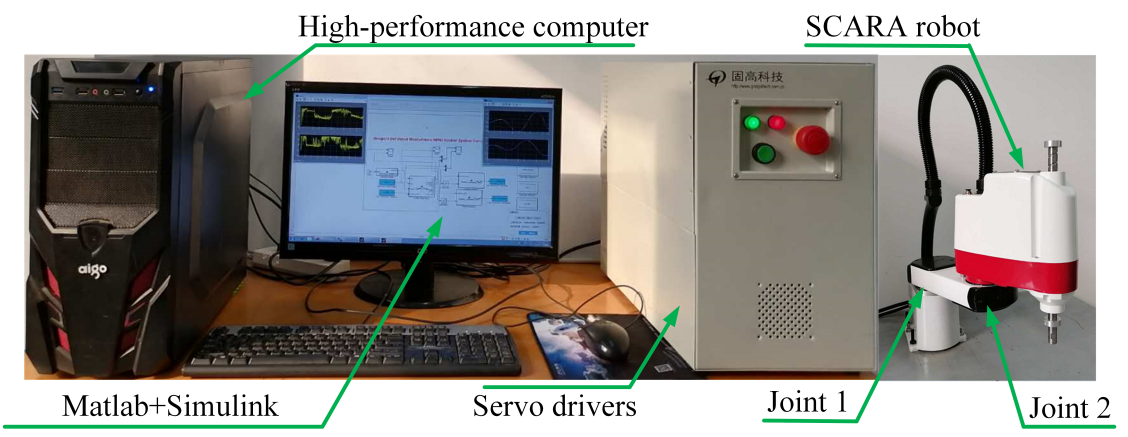

Fig. 6 The experimental robot setup.

Table 3 Controller parameters selection

\begin{tabular}{lc}
\hline Controllers & Parameters \\
\hline \multirow{2}{*}{ AFTC-ISM } & $\kappa=0.01, r=0.5, \alpha=\operatorname{diag}\{30,10\}, \vartheta_{0}=0.7, \vartheta_{1}=0.7$ \\
& $\beta=\operatorname{diag}\{70,40\}, \gamma_{1}=0.5, \bar{\gamma}_{1}=0.09, \bar{\gamma}_{2}=0.2, \gamma_{0}=0.65$ \\
\hline \multirow{2}{*}{ FIBC } & $K_{1}=K_{2}=K_{3}=\operatorname{diag}\{8,8\}, \rho_{i 0}=[2 ; 2], \alpha=0.8, \varpi_{0}=[0 ; 0]$ \\
& $K_{4}=K_{5}=K_{6}=\operatorname{diag}\{1,1\}, v_{i}=0.01, \varepsilon=\varsigma=0.05$ \\
\hline \multirow{2}{*}{ ANFTSMC } & $\alpha=1.1, \beta=5 / 3, k_{1}=\operatorname{diag}\{10,30\}, k_{2}=\operatorname{diag}\{2,1\}$ \\
& $\Lambda=\operatorname{diag}\{150,100\}, \lambda_{0}=\lambda_{1}=\lambda_{2}=0.7, \eta=50$ \\
\hline
\end{tabular}

In this experimental comparisons, the sample period is chosen as $t_{s}=2 \mathrm{~ms}$. The initial states are $q=[0 ; 0]$ (rad) and the desired trajectories are

$$
q_{d}=\left[-\frac{\pi}{4} \sin \left(0.5 t+\frac{\pi}{2}\right), \frac{\pi}{4} \sin \left(0.5 t+\frac{\pi}{2}\right)\right]^{T}
$$

For a fair comparison, the same health condition (58) has been added in the following experimental comparisons. Fig. 7 gives an real tracking from real position to the desired trajectories. Fig. 8 illustrate the errors in position tracking of AFTC-ISM, ANFTSMC and FIBC with their zoomed plots; while Fig. 9 gives the requested control of AFTC-ISM, ANFTSMC and FIBC. As shown in Fig. 8, the proposed approach reveals rapid transient response and 


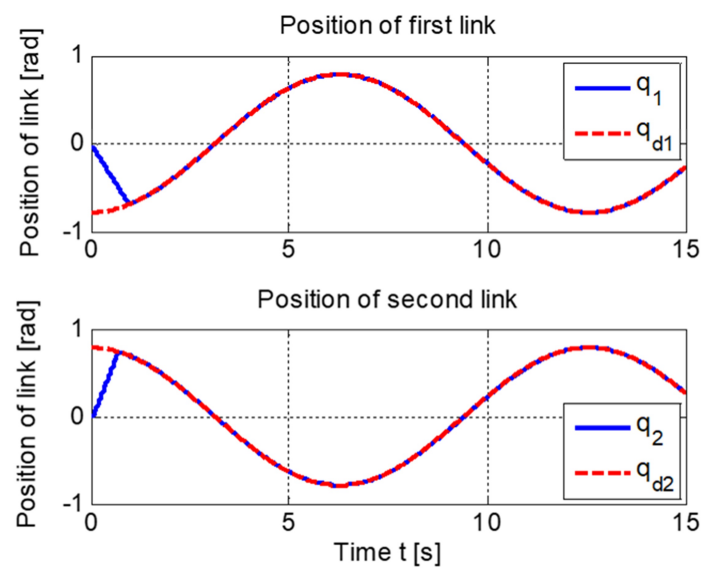

Fig. 7 Positions of AFTC-ISM.
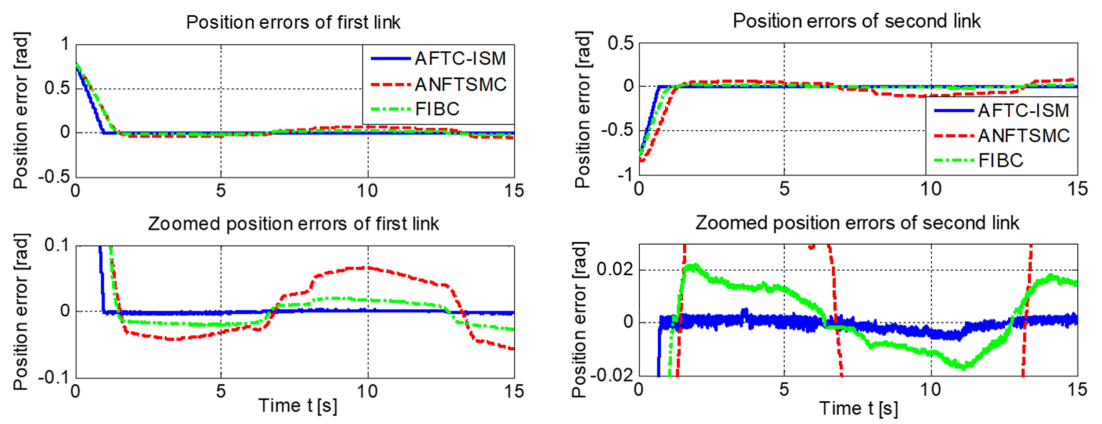

Fig. 8 Position tracking errors of ANFTSMC, FIBC and AFTC-ISM.
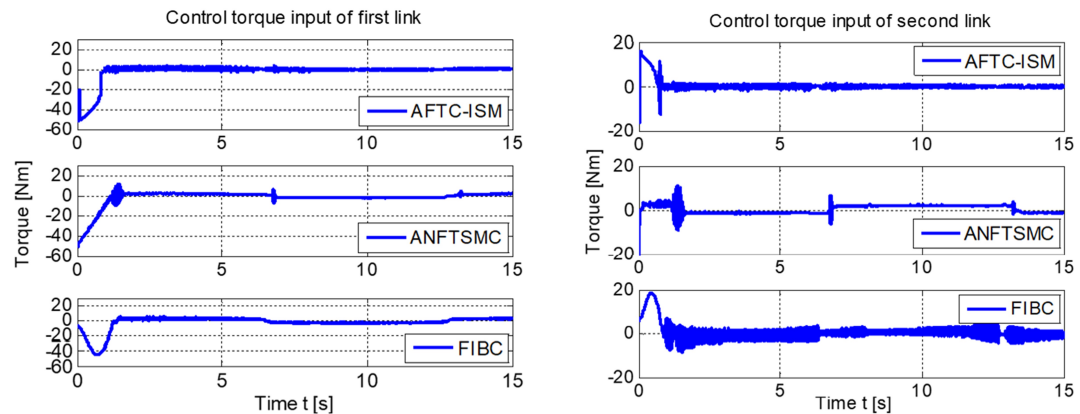

Fig. 9 Control torque input of ANFTSMC, FIBC and AFTC-ISM.

higher steady-state tracking precision than FIBC and ANFTSMC. From the zoomed plots of Fig. 8, the steady-state tracking errors of two joints are stabled at $5 \times 10^{-3}(\mathrm{rad})$ and $1 \times 10^{-3}(\mathrm{rad})$, respectively. From Fig. 9, the advanced tracking performance of the proposed AFTC-ISM in transient and steadystate does not utilizing the excessive control torque input. Upon the above 
comparisons shown in Figs. 8 and 9, the experimental results further show a fact that the proposed approach gains fast transient speed and high steadystate tracking precision than FIBC and ANFTSMC, which is also completed by a simple control structure.

Furthermore, the quantitative comparisons similar to (66) and (67) have been accomplished in Table 4 to show the the improved tracking performance of the proposed AFTC-ISM.

Table 4 Comparison of control performance

\begin{tabular}{lcc}
\hline Controller & $\bar{E}_{p}$ & $\bar{E}_{\tau}$ \\
\hline AFTC-ISM & 0.1599 & 1.0151 \\
ANFTSMC & 0.489 & 2.4653 \\
FIBC & 0.382 & 3.1915 \\
\hline
\end{tabular}

Similarly, in Table 4 the position and velocity tracking errors with their control efforts have been calculated in range $t>3 \mathrm{~s}$ at the beginning of the experimental comparisons. Obviously, the proposed approach also shows the minimum position and velocity tracking errors than FIBC and ANFTSMC. Such favour comparison results of the proposed approach also does not utilized the excessive control efforts.

Accordingly, from the results of Figures 1-9 and Tables 2 and 4, the proposed AFTC-ISM still reveals the enhanced tracking performance for uncertain robot manipulators subject to AEFs in both simulation and experimental comparisons.

\section{Conclusion}

In this paper, an adaptive FTC featuring with strong robustness, chatteringrestraining, rapid transient response, high steady-state precision and simple control structure, has been proposed for uncertain robot manipulators. The simulation and experimental comparisons gain the fact that the proposed approach greatly improves the steady-state tracking precision than other controllers observed from Figs. 1-7 and Table 2. Meanwhile, such favour tracking performance does not uses the excessive control input torque. Moreover, the proposed AFTC-ISM removes the singularity and algebraic loop of SMC for uncertain robot manipulators; the reaching phase is removed by the proposed approach for chattering-restraining SMC. Then, the developed control strategy offers an alternative approach for improving the design of uncertain robot manipulators for fault-tolerant tracking problem.

Acknowledgements This work was supported in part by Natural Science Foundation of Shaanxi Province (Grand No. 2020JQ-847) and in part by Scientific Research Program Funded by Shaanxi Provincial Education Department (Program No. 20JK0914) 


\section{Data Availability}

The datasets generated during and/or analysed during the current study are available from the corresponding author on reasonable request.

\section{Conflict of interest}

The authors declare that they have no conflict of interest.

\section{References}

1. Yu. H., Shen J. H., Joos K. M., and Simaan N. Calibration and integration of b-mode optical coherence tomography for assistive control in robotic microsurgery. IEEE/ASME Trans. Mechatronics, 21(6):2613-2623, 2016.

2. Xiao B., Yin S., and O. Kaynak. Tracking control of robotic manipulators with uncertain kinematics and dynamics. IEEE Trans. Ind. Electron., 63(10):6439-6449, 2016.

3. Lee J. Y., Jin M. L., and Chang P. H. Variable pid gain tuning method using backstepping control with time-delay estimation and nonlinear damping. IEEE Trans. Ind. Electron., 61(12):6975-6985, 2014.

4. Jinoh Lee, Pyung Hun Chang, and Rodrigo S Jamisola. Relative impedance control for dual-arm robots performing asymmetric bimanual tasks. IEEE Trans. Ind. Electron. 61(7):3786-3796, 2013.

5. Islam S. and Liu X. P. Robust sliding mode control for robot manipulators. IEEE Trans. Ind. Electron., 58(6):2444-2453, 2010.

6. Wang J., Zhou Y. D., Bao Y. L., Kim H. H., and Lee M. C. Trajectory tracking control using fractional-order terminal sliding mode control with sliding perturbation observer for a 7-dof robot manipulator. IEEE/ASME Trans. Mechatronics, 25(4):1886-1893, 2020 .

7. Jin M. L., Kang S. H., Chang P., and Lee J. Robust control of robot manipulators using inclusive and enhanced time delay control. IEEE/ASME Trans. Mechatronics, 22(5):2141-2152, 2017.

8. Mien V. An enhanced robust fault tolerant control based on an adaptive fuzzy pid-nonsingular fast terminal sliding mode control for uncertain nonlinear systems. IEEE/ASME Trans. Mechatronics, 23(3):1362-1371, 2018.

9. Khanzadeh A. and Pourgholi M. Fixed-time sliding mode controller design for synchronization of complex dynamical networks. Nonlinear Dynamics, 88(4):2637-2649, 2017.

10. Mien V., Ge S. Z., and Ren H. L. Robust fault-tolerant control for a class of secondorder nonlinear systems using an adaptive third-order sliding mode control. IEEE Trans. Syst., Man, Cybern. Sys., 47(2):221-228, 2016.

11. Huang H. F., He W., Li J. S., Xu B., Yang C. G., and Zhang W. C. Disturbance observer-based fault-tolerant control for robotic systems with guaranteed prescribed performance. IEEE Trans. Cybern., 2020.

12. Mien Van, Hee-Jun Kang, Young-Soo Suh, and Kyoo-Sik Shin. A robust fault diagnosis and accommodation scheme for robot manipulators. Int. J. Control, Autom. Syst., 11(2):377-388, 2013.

13. He X., Wang Z. D., Qin L. G., and D. H. Zhou. Active fault-tolerant control for an internet-based networked three-tank system. IEEE Trans. Control Syst. Technol., 24(6):2150-2157, 2016

14. Mien V., Mavrovouniotis M., and Ge S. Z. An adaptive backstepping nonsingular fast terminal sliding mode control for robust fault tolerant control of robot manipulators. IEEE Trans. Syst., Man, Cybern. Sys., 49(7):1448-1458, 2018. 
15. Zhang L. Y., Liu H., Tang D. F., Hou Y. L., and Wang Y. M. Adaptive fixed-time fault-tolerant tracking control and its application for robot manipulators. IEEE Trans. Ind. Electron.

16. Xiao B., Hu Q. L., and Zhang Y. M. Adaptive sliding mode fault tolerant attitude tracking control for flexible spacecraft under actuator saturation. IEEE Trans. Control Syst. Technol., 20(6):1605-1612, 2011.

17. Meng Q., Zhang T., Gao X., and Song J. Y. Adaptive sliding mode fault-tolerant control of the uncertain stewart platform based on offline multibody dynamics. IEEE/ASME Trans. Mechatronics, 19(3):882-894, 2013.

18. Mien V., Mavrovouniotis M., and Ge S. Z. An adaptive backstepping nonsingular fast terminal sliding mode control for robust fault tolerant control of robot manipulators. IEEE Trans. Syst., Man, Cybern. Sys., 49(7):1448-1458, 2018.

19. Mien V., Ge S. Z., and Ren H. L. Finite time fault tolerant control for robot manipulators using time delay estimation and continuous nonsingular fast terminal sliding mode control. IEEE Trans. Cyber., 47(7):1681-1693, 2016.

20. W-H Zhu. Comments on" robust tracking control for rigid robotic manipulators". IEEE Transactions on Automatic Control, 45(8):1577-1580, 2000.

21. Cao W. J. and Xu J. X. Nonlinear integral-type sliding surface for both matched and unmatched uncertain systems. IEEE Trans. Automat. Contr., 49(8):1355-1360, 2004.

22. Wang Y. Y., Xia Y. Q., Li H. Y., and Zhou P. F. A new integral sliding mode design method for nonlinear stochastic systems. Automatica, 90:304-309, 2018.

23. Niu Y. G., Ho D., and Lam J. Robust integral sliding mode control for uncertain stochastic systems with time-varying delay. Automatica, 41(5):873-880, 2005.

24. Jiang B. P., Karimi H. R., Kao Y. G., and Gao C. C. A novel robust fuzzy integral sliding mode control for nonlinear semi-markovian jump t-s fuzzy systems. IEEE Trans. Fuzzy Syst., 26(6):3594-3604, 2018.

25. Lee J. Y., Chang P. H., and Jin M. L. Adaptive integral sliding mode control with timedelay estimation for robot manipulators. IEEE Trans. Ind. Electron., 64(8):6796-6804, 2017.

26. Zhang L. Y., Liu L. Z., Wang Z., and Xia Y. Q. Continuous finite-time control for uncertain robot manipulators with integral sliding mode. IET Control Theory Appli., 12(11):1621-1627, 2018.

27. Ferrara A. and Incremona G. P. Design of an integral suboptimal second-order sliding mode controller for the robust motion control of robot manipulators. IEEE Trans. Control Syst. Technol., 23(6):2316-2325, 2015.

28. Wang L., Du H. B., Zhang W. J., Wu D., and Zhu W. W. Implementation of integral fixed-time sliding mode controller for speed regulation of pmsm servo system. Nonlinear Dynamics, 102(1):185-196, 2020.

29. Qin J. H., Ma Q. C., Gao H. J., and Zheng W. X. Fault-tolerant cooperative tracking control via integral sliding mode control technique. IEEE/ASME Trans. Mechatron., 23(1):342-351, 2017.

30. Hamayun M. T., Edwards C., and Alwi H. Design and analysis of an integral sliding mode fault-tolerant control scheme. IEEE Trans. Automat. Contr., 57(7):1783-1789, 2011.

31. Li-Ying Hao, Ju H Park, and Dan Ye. Integral sliding mode fault-tolerant control for uncertain linear systems over networks with signals quantization. IEEE Trans. Neural Netw. Learn. Syst., 28(9):2088-2100, 2016.

32. Chen C. C., Xu S. D., and Liang Y. W. Study of nonlinear integral sliding mode fault-tolerant control. IEEE/ASME Trans. Mechatron., 21(2):1160-1168, 2015.

33. Shen Q, Wang D. W., Zhu S. Q., and Poh E. K. Integral-type sliding mode faulttolerant control for attitude stabilization of spacecraft. IEEE Trans. Control Syst. Technol., 23(3):1131-1138, 2014.

34. Mien V. and Ge S. Z. Adaptive fuzzy integral sliding-mode control for robust faulttolerant control of robot manipulators with disturbance observer. IEEE Trans. Fuzzy Syst., 29(5):1284-1296, 2020.

35. Yu H., Xie T. T., Paszczynski S., and Wilamowski B. M. Advantages of radial basis function networks for dynamic system design. IEEE Trans. Ind. Electron., 58(12):54385450, 2011. 
36. Lijie Wang, Michael V Basin, Hongyi Li, and Renquan Lu. Observer-based composite adaptive fuzzy control for nonstrict-feedback systems with actuator failures. IEEE Transactions on Fuzzy Systems, 26(4):2336-2347, 2017.

37. Yu J. P., Zhao L., Yu H. S., Lin C., and Dong W. J. Fuzzy finite-time command filtered control of nonlinear systems with input saturation. IEEE Trans. Cybern., 48(8):2378$2387,2017$.

38. Zhang J. X. and Yang G. H. Fuzzy adaptive output feedback control of uncertain nonlinear systems with prescribed performance. IEEE Trans. Cybern., 48(5):1342-1354, 2017.

39. Wang H. Q., Liu X. P., Li S., and Wang D. Adaptive neural output-feedback control for a class of nonlower triangular nonlinear systems with unmodeled dynamics. IEEE Trans. Neural Netw., 29(8):3658-3668, 2017.

40. Mobayen S. An adaptive chattering-free pid sliding mode control based on dynamic sliding manifolds for a class of uncertain nonlinear systems. Nonlinear Dynamics, 82(1):53$60,2015$.

41. Mobayen S. An adaptive fast terminal sliding mode control combined with global sliding mode scheme for tracking control of uncertain nonlinear third-order systems. Nonlinear Dynamics, 82(1):599-610, 2015.

42. Shen G. H., Xia Y. Q., Zhang J. H., and Cui B. Adaptive fixed-time trajectory tracking control for mars entry vehicle. Nonlinear Dynamics, 102(4):2687-2698, 2020.

43. Huang Y. J., Kuo T. C., and Chang S. H. Adaptive sliding-mode control for nonlinearsystems with uncertain parameters. IEEE Trans. Syst. Man Cybern. B Cybern., 38(2):534-539, 2008.

44. Plestan F., Shtessel Y., V. Brégeault, and Poznyak A. Sliding mode control with gain adaptationapplication to an electropneumatic actuator. Control Eng. Prac., 21(5):679$688,2013$.

45. Neila M. B. R. and Tarak D. Adaptive terminal sliding mode control for rigid robotic manipulators. Int. J. Auto. Comput., 8(2):215-220, 2011.

46. Baek J., Jin M. L., and Han S. A new adaptive sliding-mode control scheme for application to robot manipulators. IEEE Trans. Ind. Electron., 63(6):3628-3637, 2016.

47. Chiu C. S. Derivative and integral terminal sliding mode control for a class of mimo nonlinear systems. Automatica, 48(2):316-326, 2012.

48. Feng Y., Yu X. H., and Man Z. H. Non-singular terminal sliding mode control of rigid manipulators. Automatica, 38(12):2159-2167, 2002.

49. Liu L. Z., Zhang L. Y., Wang Y. M., and Hou Y. L. A novel robust fixed-time faulttolerant tracking control of uncertain robot manipulators. IET Control Theory App., 2020.

50. Wang L. Y., Chai T. Y., and Zhai L. F. Neural-network-based terminal sliding-mode control of robotic manipulators including actuator dynamics. IEEE Trans. Ind. Electron., 56(9):3296-3304, 2009.

51. Boukattaya M., Mezghani M., and Damak T. Adaptive nonsingular fast terminal slidingmode control for the tracking problem of uncertain dynamical systems. ISA Trans. $77: 1-19,2018$.

52. Smaeilzadeh S. M. and Golestani M. Finite-time fault-tolerant adaptive robust control for a class of uncertain non-linear systems with saturation constraints using integral backstepping approach. IET Control Theory App., 12(15):2109-2117, 2018. 